\title{
Pseudoaneurysm with arteriovenous fistula of the prostate after pelvic trauma: Ultrasound imaging
}

\author{
Andrea B. Galosi, Camilla Capretti, Luca Leone, Marco Tiroli, Daniele Cantoro, Massimo Polito \\ Institute of Urology, Department of Specialized Clinical Sciences and Odontostomatology, Marche Polytechnic University, \\ Azienda Ospedaliero-Universitaria, Ancona, Italy.
}

\begin{abstract}
Summary Pseudoaneurysm (PA) associated with an arteriovenous fistula (AVF) of the internal pudendal artery branches are very uncommon. We report a case of post-traumatic PA with AVF connected to Santorini plexus. Diagnosis was made with trans-rectal ultrasound (TRUS) after recurrent hematuria. TRUS reported a 1.7 $\times 1.4 \times 1.5 \mathrm{~cm}$ anechoic area, on anterior prostate apex close to Santorini plexus. The use of color Doppler in this area revealed high flow velocity that was indicative for AVF. The feeding artery was a distal branch of the left pudenda artery. After selective embolization was observed complete occlusion of the feeding branches and disappearance of PA with AVF. Prostate PA with concomitant symptomatic AVF detected with TRUS has not yet described in literature after pelvic trauma and represents complex diagnostic challenges. This case report suggests that the use of TURS and color Doppler can provide an important diagnostic and follow-up to address the clinical suspicion of occult vascular injuries using a noninvasive approach.
\end{abstract}

KEY WORDS: Prostate; Pseudoaneurysm; Arteriovenous fistula; Transrectal ultrasonography; Pelvic trauma.

Submitted 15 November 2016; Accepted 15 December 2016

\section{INTRODUCTION}

Prostate Pseudoaneurysms (PA) associated with arteriovenous fistula (AVF) are very uncommon (1) and observed after prostatic surgery. To our knowledge there are no cases described of post-traumatic PA with AVF detected by trans-rectal ultrasound (TRUS); in literature, the only case detected by TRUS is secondary to extraperitoneal laparoscopic radical prostatectomy (2).

$\mathrm{PA}$, also called false aneurysm, is a perfused sac communicating with an arterial wall disruption. It is delimited by avventitia or media layer or soft-tissue surrounding the artery (3). PA develops from injury or disease (infection or inflammation) of the vessel wall and it may lead to complications such as thrombosis, embolism, rupture or infection (4-5). Also AVF could be considered a complication of PA. This vascular lesion is slow-growing and clinical manifestations are delayed after causing trauma. Therefore complications could be later and diagnosis may be difficult. Only angiography or computed tomography $(\mathrm{CT})$ angiography are the best diagnostic imaging (6). Ultrasonography (US) with color Doppler has high sensibility and specificity in PA (5), however its application in the prostate after pelvic trauma has not been reported yet. TRUS represents a faster and available method to evaluate prostatic disease.

We report a case of massive hematuria due to post-traumatic pesudoaneurysm of the prostate with arteriovenous fistula with Santorini plexus.

\section{Case report}

A 36-years-old man was referred to emergency department for hematuria after blunt pelvic trauma in a car crash. Contrast enhanced abdominal CT and the retrograde urethrogram showed bladder extraperitoneal injury, pelvic bone fracture and bulbar urethral lesion (Figure 1A, B, C).

Figure 1.

A. Contrast computed tomography (CT), axial scan: left obturator internal fossa hematoma compressing and deviating bladder to the right.

B. Contrast computed tomography (CT), coronal scan: bladder injury in the anterior and left lateral wall.

C. Retrograde urethrogram: bladder injury in the anterior and left lateral wall.

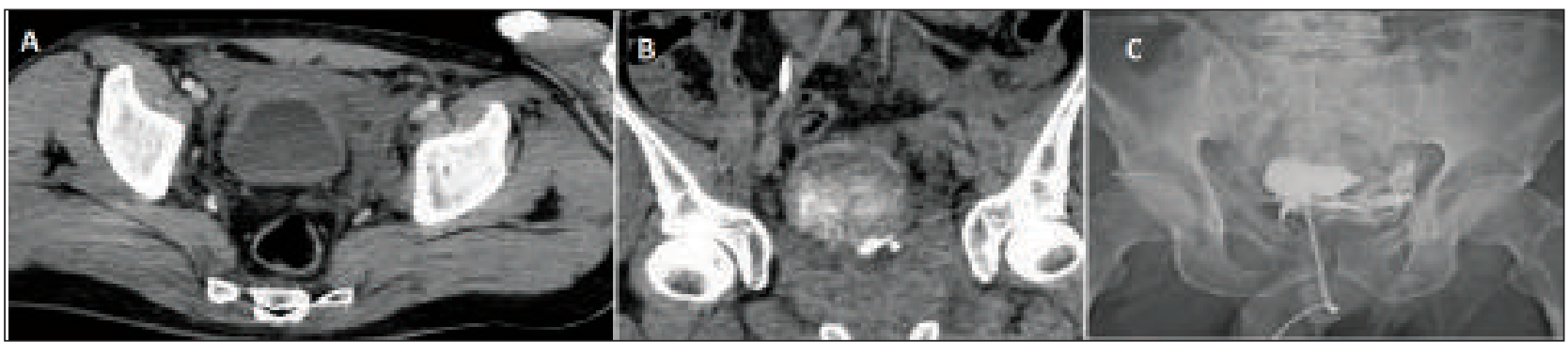

No conflict of interest declared. 


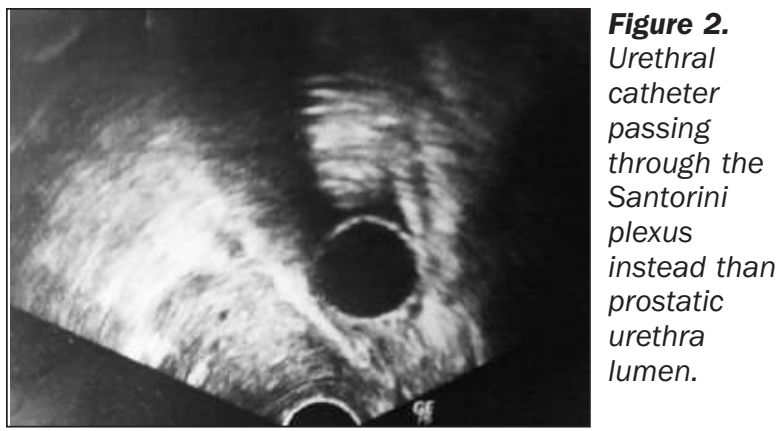

In the emergency room a bladder catheter was already placed. Patient was treated conservatively and pelvic hematoma disappeared. He underwent neurosurgical procedure for brain hematoma. Anti-thrombotic prophylaxis with low molecular weight heparin was prescribed. Patient was discharged and transurethral catheter was planned to be removed after 30 days. Soon after normal retrograde cystography and urethrogram, a massive urethral bleeding developed. Bleeding stopped immediately after transurethral insertion of a new catheter. CT scan and TRUS showed that the urethral catheter was placed in the bladder, but passing through the Santorini plexus instead of the prostatic urethra lumen (Figure 2). Periprostatic tissue were normal at that time.

Forty-six days after trauma, open transvesical surgical realignment was performed removing the catheter through the Santorini plexus and placing a trans-urethral and suprapubic catheters. The urethral catheter was removed in $15^{\text {th }}$ post-operative day (60 days after trau$\mathrm{ma}$ ), after retrograde urethrogram and recovery of spontaneous urinary stream; 8 hours later, a massive hematuria developed again spontaneously and stopped with placement of a urethral catheter.

TRUS was performed, reporting a $1.7 \times 1.4 \times 1.5 \mathrm{~cm}$ anechoic area, on the left side of prostate apex, located between the urethra (with catheter) and the Santorini plexus (Figure 3A). The use of color Doppler in this area revealed high flow velocity that was suggestive for AVF (Figure 4A).

Angiography and CT Angiography confirmed a contrastenhanced lesion (about $1 \mathrm{~cm}$ ) on the left side of prostate base, behind the pubic bone, with early communication

\section{Figure 3.}

A. Trans-rectal ultrasonography: a $1.7 \times 1.4 \times 1.5 \mathrm{~cm}$ anechogenic area (arrow) on the left side of prostate capsule, adjacent Santorini plexus. U: catheter.

B. Trans-rectal ultrasonography: absence of the anechoic area after embolization (arrow).

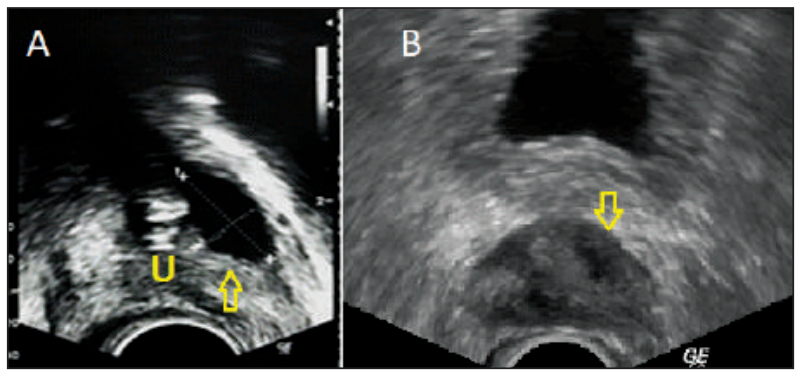

Figure 4.

A. Trans-rectal ultrasonography with Color Doppler: high flow velocity that was indicative for AVF.

B. Angiography CT scan: contrast-enhanced lesion (about $1 \mathrm{~cm}$ ) on the left side of prostate base, behind the pubic bone, with an early communication with Santorini plexus.
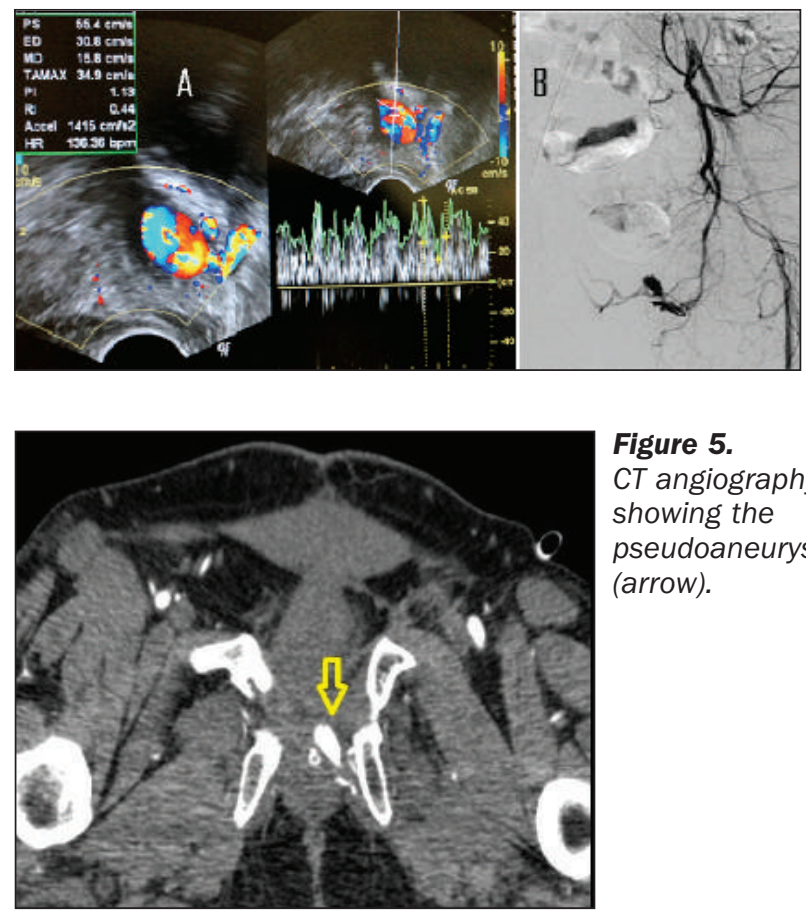

Figure 5. CT angiography showing the pseudoaneurysm (arrow).

with Santorini plexus (Figures 4B, 5); the feeding artery was a distal branch of the left pudendal artery. Embolization of the vascular anomaly was carried out by using minicoils.

Subsequent angiograms showed an almost complete occlusion of the feeding branches and no persistence of AVF. Also TRUS was used for post-embolization control, showing the absence of the anechoic area (Figure 3B); the color Doppler showed the absence high flux signal, indicating the closure of the communication with Santorini plexus. After then, the catheters were removed and the outcome was uneventful. After 8 months of follow-up the patients is doing well.

\section{Discussion}

Prostate PA with AVF detected with TRUS has not yet described in literature, in particular after a pelvic trauma. Other cases described in literature are linked to urological surgery (radical prostatectomy (6-7), transurethral resection of prostate (2), transvesical prostatectomy (1) and holmium laser enucleation (8)). We can observe that false passage of the catheter through the Santorini plexus could be considered a iatrogenic cause of the PA. Prolonged antithrombotic prophylaxis may have a role to avoid thrombosis in the PA with spontaneous resolution.

PA developed 2 months after initial trauma. Late hematuria was the first sign of PA in all cases reported by others authors, such as our patient; the persistence of hematuria (after surgical realignment) allowed us to continue diagnostic process 
For our experience, TRUS has been a valuable diagnostic methodic for the first detection; it is readily available, inexpensive and fast (9). In others anatomical sites, US has been reported to have high sensitivity (94\%) and high specificity (97\%) in the detection of PA (5).

Gray-scale TRUS can demonstrate the PA as a hypoechoic cystic. This anechoic lesion should be considered as differential diagnosis with cystic lesion of the prostate. The use of color Doppler is helpful for the diagnosis and necessary for a differential diagnosis with prostatic cyst (10). TRUS can assess size of the PA sac, however the connection of the sac to the artery was not evident in our case. Furthermore, any compartments of the PA could be observed (11).

Doppler presentation of PA with AVF is characterized by a typical swirling motion called the "yin-yang sign" within a cystic structure: it indicates bidirectional flow due to swirling of blood (12) as shown in Figure 4A.

Angiography shows a real-time hemodynamic assessment of a selective vascular bed (including identification of collateral vessels); furthermore, angiography has also a therapeutic role. In our experience angiography confirmed the PA with early venous communication, feeding by internal left pudendal artery distal branches.

After the embolization we performed a TRUS with color Doppler to confirm the resolution of the PA and the closure of the AVF: TRUS has proved to be an important diagnostic tool also in the follow-up after the treatment of PA with AVF.

\section{Conclusions}

Prostate pseudoaneurysm with concomitant symptomatic arteriovenous fistula detected with TRUS has not yet described in literature after pelvic trauma. Delayed presentation of pseudoaneurysm represents a complex challenges in diagnosis and treatment. This case report supports transrectal ultrasonography with color Doppler in differential diagnosis and in the follow-up, using a noninvasive and inexpensive approach.

\section{REFERENCES}

1. Dell'Atti L, Galeotti R. Pseudoaneurysm secondary to transvesical prostatectomy. Indian J Urol. 2016; 32: 164165.

2. Celtikci P, Ergun O, Tatar IG, et al. Superselective Arterial Embolization of Pseudoaneurysm and Arteriovenous Fistula Caused by Transurethral Resection of the Prostate. Pol J Radiol. 2014; 79:352-5.

3. Schwartz LB, Clark ET, Gewetz BL. Anastomotic and other pseudoaneurysms. In: Rutherford RB, ed. Vascular Surger. 5th ed. Philadelphia,PA: Saunders. 2000; 752-763.

4. La Perna L, Olin JW, Goines D, et al. Ultrasound-guided thrombin injection for the treatment of postcatheterization pseudoaneurysms. Circulation 2000; 102: 2391-2395.

5. Morgan R, Belli A. Current treatment methods for postcatheterization pseudoaneuryms. J Vasc Intervent Radiol. 2003; 14:697-710.

6. Jeong $\mathrm{CW}$, Park $\mathrm{YH}, \mathrm{Ku} \mathrm{JH}$, et al. Minimally invasive management of postoperative bleeding after radical prostatectomy: Transarterial embolization. J Endourol. 2010; 24:1529-33.

7. Lopes RI, Mitre AI, Rocha FT, et al. Case report: late recurrent hematuria following laparoscopic radical prostatectomy may predict internal pudendal artery pseudoaneurysm and arteriovenous fistula. J Endourol. 2009; 23:297-9.

8. Asimakopoulus AD, Dutto L, Preziosi P, et al. Holmium laser enucleation of the prostate and iatrogenic arteriovenous fistula treated by superselective arterial embolization. Case Rep Urol. 2016; 2016:4918081.

(9. Saad NE, Saad WE, Davies MG, et al. Pseudoaneurysms and the Role of Minimally Invasive Techniques in Their Management. Radiographics. 2005; 25 (Suppl 1):S173-89.

10. Galosi AB, Montironi R, Fabiani A, et al. Cystic lesions of the prostate gland: an ultrasound classification with pathological correlation. J Urol. 2009; 181:647-57.

11. Kruger K, Zahringer M, Sohngen FD, et al. Femoral pseudoaneurysms: management with percutaneous thrombin injections-success and effects on systemic coagulation. Radiology 2003; 226:452-458.

12. Kapoor BS, Haddad HL, Saddekni S, Lockhart ME. Diagnosis and management of pseudoaneurysms: an update. Curr Probl Diagn Radiol. 2009; 38:170-188.

\author{
Correspondence \\ Andrea B. Galosi, MD (Corresponding Author) \\ galosiab@yahoo.it \\ Camilla Capretti, MD \\ camilla.capretti2@gmail.com \\ Marco Tiroli, MD \\ Daniele Cantoro, MD \\ Luca Leone, MD \\ Matteo Tallè, MD \\ Matteo Cevenini, MD \\ Massimo Polito, MD \\ Scuola di Specializzazione in Urologia \\ Università Politecnica delle Marche \\ Clinica Urologica, Azienda Ospedaliero-Universitaria Ospedali Riuniti Ancona \\ Via Conca 71, 61100 Torrette di Ancona, Italy
}

